\title{
Religiöse Erziehung und Moralentwicklung: Der Einfluss einer religiös geprägten Kindheit auf die moralische Orientierung und Kompetenz
}

\author{
Asli Akin \\ ORCID: https://orcid.org/0000-0001-7558-0140 \\ (MSB Medical School Berlin - Hochschule für Gesundheit und Medizin, asli.akin@gmx.de)
}

\section{Einleitung}

Verschiedene moralpsychologische Untersuchungen haben gezeigt, dass dogmatische Religiosität die Entwicklung von Moralkompetenz hemmen kann (Lupu 2009; Saeidi-Parvaneh 2011). Aber man weiß noch wenig darüber, wie sich ein religiöses Elternhaus auf die Entwicklung der Moralkompetenz der nachfolgenden Generation auswirkt. Der vorliegende Artikel soll dieser Frage nachgehen und die ersten empirischen Erkenntnisse zum Verhältnis von religiöser Erziehung und Moralentwicklung vorstellen.

Moralkompetenz gilt als einer der beiden Aspekte des moralischen Verhaltens. Sie ist definiert als „die Fähigkeit, Probleme und Konflikte auf der Grundlage von universellen Moralprinzipien durch Denken und Diskussion zu lösen, statt durch Gewalt, Betrug und Macht" (Lind 2015, 53). Der zweite Aspekt bezieht sich auf die moralischen Orientierungen. Während die moralischen Orientierungen nach Lind als angeboren gelten, kann und muss Moralkompetenz entwickelt und erlernt werden. Dass insbesondere Gelegenheiten zur Anwendung dieser Fähigkeit in Bildungseinrichtungen eine positive Kompetenzentwicklung begünstigen, haben Lind und seine Arbeitsgruppe an der Universität Konstanz in verschiedenen Studien erwiesen (Lind 2000, 2003; Lupu 2009; Saeidi-Parvaneh 2011; Schillinger 2006).

In der bisherigen Moralkompetenz-Forschung bleibt jedoch eine Frage unbeachtet: Welche Bedeutung haben primäre frühkindliche Erziehungserfahrungen für die Entwicklung der Moralkompetenz?

Bei der Moralerziehung von Kindern spielen insbesondere Religionen eine nachweisbare und geläufige Rolle. Dass die religiöse Erziehung trotz vorherrschender Säkularisierungsauffassung in der westlichen Welt weit verbreitet ist, konnte unter anderem in einer Studie des Instituts für Demoskopie Allensbach bestätigt werden. Im 
Jahr 2006 wurden 1000 Probanden über die Wichtigkeit einer religiösen Erziehung für Kinder befragt. Während rund 40 Prozent der Befragten eine religiöse Erziehung als wichtig erachteten, äußerten sich dem Gegenüber vier Prozent der Befragten gegen eine religiöse Erziehung (Haumann 2006).

Im Folgenden soll die weit verbreitete und gleichzeitig stark umstrittene religiöse und im Besonderen dogmatisch religiöse Erziehung eines Kindes in seiner Bedeutung für die moralische Entwicklung untersucht werden. Der Artikel fasst die Ergebnisse einer quantitativen Befragung von 122 Studierenden aus dem Jahr 2018 zusammen, in der der Einfluss einer religiösen frühkindlichen Erziehung auf die Entwicklung der moralischen Orientierung und Kompetenz erforscht wurde. In der Studie wurde auf das Ausmaß dogmatischer Religiosität fokussiert, die durch die strikte und verbindliche Auslebung einer bestimmten Konfession gekennzeichnet ist. Diese ist von persönlicher Religiosität oder Spiritualität deutlich abzugrenzen, bei der Gläubige nicht an die Dogmen einer bestimmten Religionsgemeinschaft glauben, sondern an einen persönlichen, überkonfessionellen Gott.

In der Untersuchung wurden die zwei nachstehend aufgeführten Hypothesen überprüft:

Hypothese 1: Studierende, die eine dogmatisch religiöse Erziehung in der frühen Kindheit erlebten, weisen eine geringere Moralkompetenz auf als Studierende ohne diese Erfahrung. Die dogmatische Auslebung einer Religion wurde bereits als hemmender Faktor für die moralische Kompetenz erwiesen. Die Studien von Lupu (2009) und Saeidi-Parvaneh (2011) zeigen die statistisch signifikante negative Wirkung der dogmatischen Religiosität auf die Höhe der moralischen Kompetenz. Die beiden Forscher entdeckten bedeutend große Moralkompetenz-Differenzen von 4.2 bis 9.18 C-Punkten zwischen dogmatisch religiösen und nicht religiösen Studierenden. Daran anlehnend wurde angenommen, dass auch ein dogmatisch religiöses Elternhaus die Entwicklung der moralischen Kompetenz hemmt und dass diese Hemmung selbst im jungen Erwachsenenalter noch nachweisbar ist. Die frühkindliche Erziehungserfahrung wird basierend auf sozialpsychologischen und psychoanalytischen Theorien (vgl. Adorno 1950; Fromm 2014; Gruen 2000) als ein bedeutender Einflussfaktor für die Moralkompetenzentwicklung vermutet. Diese implizieren, dass die autoritäre Vermittlung religiöser Dogmen zur Ausbildung einer Identität führen kann, die sich an dem Willen der Religion orientiert und ein autonomes Denken verhindert, sodass moralische Prinzipien nicht hinterfragt oder diskutiert werden können.

Hypothese 2: Studierende, die eine dogmatisch religiöse Erziehung in der frühen Kindheit erlebten, präferieren häufiger als nicht religiös erzogene Studierende niedrige (bzw. präkonventionelle) moralische Orientierungen. Der affektive Aspekt der Moral lässt sich in der Orientierung an den Stufen der Moralentwicklung nach Kohlberg (1996) abbilden. Die Präferenzhierarchie an den moralischen Orientierungen, das heißt der gleichmäßige Anstieg der Einstellungen zu 
den Argumenten der verschiedenen Typen, hat sich im interindividuellen Vergleich als relativ stabil erwiesen (Lind 2015). In verschiedenen Altersstufen, sozialen Schichten und Kulturen werden die moralischen Orientierungen an Typ eins und zwei eher abgelehnt, während die Orientierungen an Typ fünf und sechs stark akzeptiert werden. In der religionsspezifischen Moral-Forschung zeigte sich jedoch, dass dogmatisch religiöse Menschen eine stärkere Präferenz für moralische Orientierungen an Typ eins und zwei aufweisen. Unter anderem die Studien von Richards (1991), Holley (1991) und Glover (1997) bestätigen die Bevorzugung des präkonventionellen Moralniveaus bei dogmatisch religiösen Menschen und lassen einen entsprechenden Einfluss der dogmatisch religiösen Erziehung vermuten.

\section{Methode}

Insgesamt wurden 130 Studierende aus Berlin auf Basis einer Online-Studie befragt, in der sowohl dogmatisch religiös als auch nicht religiös erzogene Probanden angeworben wurden. 122 Studierende konnten in der Datenauswertung berücksichtigt werden, während sieben Probanden aufgrund unvollständiger sowie widersprüchlicher demografischer Angaben ausgeschlossen wurden. Die Befragung fand über die Umfrageplattform QuestionPro statt. Über das hochschulinterne Sona-System der Medical School Berlin sowie die Versendung des Umfrage-Links per E-Mail und über soziale Netzwerke wurden die Studienteilnehmer rekrutiert.

Es wurden Studierende aus 18 verschiedenen Studienfächern befragt, die zu neun Studienfachrichtungen zusammengefasst werden konnten (siehe Abschnitt 3). Zum Befragungszeitpunkt waren die Probanden durchschnittlich zwei Jahre Berufstätig und 22 Jahre alt, wobei die Spannweite der Berufstätigkeit von keiner bis 20 Jahren reichte und Altersstufen von 18 bis 37 Jahren vertreten waren. Von den Befragten gaben 92.68 Prozent als höchsten Bildungsabschluss das Abitur an, während 7.32 Prozent einen Hochschulabschluss bestätigten.

\subsection{Messinstrumente}

Zur differenzierten Erfassung der Moralkompetenz und der moralischen Orientierungen wurde der standardisierte und wissenschaftlich fundierte MoralischeKompetenz-Test (MKT) nach Lind (2015) eingesetzt. Die religiöse Erziehung und aktuelle Religiosität wurde mithilfe eines selbstkonstruierten Fragebogens erfasst. Trotz der seit den 90er Jahren stetig wachsenden Vielfalt an Religiositäts-Fragebögen existiert im Rahmen der bisherigen Forschung keiner, der primär die religiöse Erziehung des Befragten erfasst. Deshalb wurde der Fragebogen Religiöse Erziehung in der Kindheit und gegenwärtige Religiosität neu konstruiert und in einer Vorstudie erprobt. 


\subsubsection{Der Moralische-Kompetenz-Test nach Lind}

Im Moralische-Kompetenz-Test(MKT) werden die Testpersonen mit zwei DilemmaGeschichten konfrontiert: das Arbeiter-Dilemma und das Arzt-Dilemma. Während das Arbeiter-Dilemma von einem Gesetzesverstoß zweier Arbeiter handelt, die ein illegales Abhören der Vorgesetzten beweisen wollen, beschreibt das Arzt-Dilemma die Sterbehilfe, die ein Arzt seiner todkranken Patientin auf ihren Wunsch leistet. Nach jedem Dilemma wird zunächst das jeweilige Verhalten der Protagonisten bewertet. Im Anschluss sollen jeweils sechs Argumente für und sechs Argumente gegen das Verhalten des Protagonisten beurteilt werden. Die sechs Argumente für und gegen das Verhalten entsprechen jeweils den sechs Stufen der Moralentwicklung nach Kohlberg.

Die moralische Kompetenz offenbart sich im Bewertungsmuster der Argumentationen. Eine hohe Moralkompetenz zeigt sich darin, dass sich ein Teilnehmer bei der Bewertung der Argumente an deren moralischer Qualität orientiert. Bei einer niedrigen Moralkompetenz sind die Bewertungen der Argumente stark von der anfänglich angegebenen Meinung determiniert. Die in den Antwortmustern sichtbare Moralkompetenz wird beim MKT durch den C-Wert ausgedrückt („C“ steht für Kompetenz). Der C-Wert wird durch die Berechnung einer intraindividuellen Varianzanalyse ermittelt und kann zwischen null und 100 variieren. Nach Lind (2018) stellt ein C-Wert zwischen .0 und 4.9 keine moralische Kompetenz dar. C-Werte zwischen 5.0 und 9.9 nennt er sehr gering und C-Werte zwischen 10.0 und 19.9 gering. Erst ein C-Wert höher als 20.0 definiert Lind als ausreichend. C-Werte über 30.0 gelten für ihn als hoch.

Jede der sechs moralischen Orientierung wird im MKT durch jeweils vier Argumente repräsentiert. Für die Analysen wird für jede der sechs Orientierungstypen ein Mittelwert berechnet. Jeder Orientierungs-Mittelwert kann von -4 bis +4 reichen, wobei höhere Werte für eine höhere Akzeptanz der jeweiligen moralischen Orientierung sprechen. Die Präferenz der sechs moralischen Orientierungen unterliegt einer universell beobachtbaren Hierarchie: Während mit steigendem Orientierungstyp die Akzeptanz ansteigt, ist bei den niedrigeren Orientierungstypen eine immer stärkere Ablehnung zu beobachten.

\subsubsection{Religiöse Erziehung in der Kindheit und gegenwärtige Religiosität}

Der Fragebogen Religiöse Erziehung in der Kindheit und gegenwärtige Religiosität ermöglicht die Erfassung von zwei Aspekten: erstens die religiöse Erziehung in der Kindheit und zweitens die aktuelle Religiosität.

Der Fragebogen besteht aus 36 Items, von denen die ersten 19 die religiöse Erziehung des Probanden erfassen und die darauffolgenden 17 die aktuelle Religiosität. Beide Teile des Fragebogens erfassen jeweils vier ähnliche und dadurch vergleichbare Skalen: 


\begin{tabular}{|l|l|l|}
\hline & Religiöse Erziehung in der Kindheit & Aktuelle Religiosität \\
\hline \multirow{5}{*}{ Skala } & Allgemeine Relevanz der Religion & Allgemeine Relevanz der Religion \\
\cline { 2 - 3 } & $\begin{array}{l}\text { Vermittlung religiöser Praktiken/ } \\
\text { Rituale }\end{array}$ & $\begin{array}{l}\text { Ausführung religiöser Praktiken/ } \\
\text { Rituale }\end{array}$ \\
\cline { 2 - 3 } & dogmatisch-religiöse Wertevermittlung & $\begin{array}{l}\text { dogmatisch-religiöse } \\
\text { Wertvorstellungen }\end{array}$ \\
\cline { 2 - 3 } & Reflexionsmöglichkeiten & $\begin{array}{l}\text { Fähigkeit zur Reflexion der eigenen } \\
\text { Religion }\end{array}$ \\
& & \\
\hline
\end{tabular}

Tabelle 1.

Inhaltliche Einteilung des Fragebogens Religiöse Erziehung in der Kindheit und gegenwärtige Religiosität

Dievier zugrundeliegenden Skalen desFragebogens wurden unter Berücksichtigung, der aus der Literatur und Forschung etablierten Aspekte der Religiosität gewählt (vgl. Albani et al. 2002; Figl 2017; Huber 2009; Jakobs 2006; Maiello 2007; Meulemann 2015).

Bei der Auswertung des Fragebogens Religiöse Erziehung in der Kindheit und gegenwärtige Religiosität können jeweils drei Ausprägungstypen der religiösen Erziehung und Religiosität unterschieden werden:

- dogmatisch religiös (erzogen)

- teilweise religiös (erzogen)

- nicht religiös (erzogen)

Die Zuordnung eines Probanden zu einem der drei Typen erfolgt anhand des ItemSummenwerts. Der Item-Summenwert kann in der ersten Fragebogenhälfte maximal 76 Punkte und in der zweiten Hälfte maximal 68 Punkte betragen, wobei niedrigere Werte für eine dogmatisch religiöse Erziehung ( $<37$ Punkte) oder dogmatische Religiosität $(<$ 33 Punkte) sprechen.

Die Objektivität und Reliabilität des Fragebogens konnten vorab durch eine Pilotierung an 10 Probanden gesichert werden.

\subsection{Auswertungsmethode}

Mit dem Ziel die Auswirkung der frühkindlichen religiösen Erziehung auf die moralische Kompetenz und Orientierung zu erfassen, wurden die Daten einer Regressionsanalyse unterzogen. Zusätzliche potenzielle Einflussfaktoren - die aktuelle Religiosität und die belegten Studienrichtungen der Probanden - wurden in der Datenanalyse mitberücksichtigt, sodass letztendlich eine multiple Regressionsanalyse gerechnet werden konnte. Dabei wurde untersucht, ob die Güte der Regression durch das Hinzufügen der zusätzlichen Einflussvariablen steigt oder sinkt. Die Voraussetzungen der Regressionsanalyse in Bezug auf die Residuen (Homoskedastizität, Normalverteilung und Unkorreliertheit) und auf die Regressoren im multivariaten Fall (Unabhängigkeit) 
wurden berücksichtigt und im Voraus geprüft. Zusätzlich wurden die absoluten Messwertunterschiede zwischen dogmatisch religiös und nicht religiös erzogenen Probanden berechnet, um über die Signifikanz hinaus die absolute Effektstärke zu veranschaulichen.

Zur Prüfung der zweiten Hypothese wurde ein Mittelwertvergleich (KruskalWallis-Test) durchgeführt, um signifikante Unterschiede zwischen dogmatisch religiös, teilweise religiös und nicht religiös erzogenen Studierenden im Hinblick auf die moralischen Orientierungstypen zu ermitteln.

\section{Ergebnisse}

Die erste Hypothese, dass dogmatisch religiös erzogene Studierende eine geringere Moralkompetenz aufweisen als Studierende ohne religiöse Erziehungserfahrungen, konnte durch die statistische Untersuchung der Daten bestätigt werden. Zwischen dem Ausmaß der dogmatisch religiösen Erziehung im Kindesalter und der aktuellen Moralkompetenz wurde eine sehr hohe Korrelation von $r=.732$ ermittelt $(p<.001)$.

Durch die Berechnung einer Regressionsanalyse konnte die Art der Beziehung zwischen C-Wert-Höhe und dogmatisch religiöser Erziehungserfahrung genauer quantifiziert werden. Die dogmatisch religiöse Erziehung in der Kindheit erwies sich als höchstsignifikanter Prädiktor für die Höhe der Moralkompetenz ( $T=11.76, p<.001) .{ }^{1}$

Um diesen bedeutenden Zusammenhang zwischen dogmatisch religiöser Erziehung und Moralkompetenz unabhängig von anderen Einflussgrößen annehmen zu können, wurden weitere potenziell intervenierende Variablen statistisch berücksichtigt. Dazu wurden unter anderem das Alter, der höchste Bildungsabschluss, die Länge der bisherigen Berufstätigkeit und die aktuelle Religiosität in ihrem Einfluss auf die C-Werte untersucht. Die ermittelten Regressionsparameter waren alle vier nicht signifikant $(p>$ .05) und konnten somit die Moralkompetenz-Differenzen der Probanden nicht erklären. Auch das Erklärungspotenzial der Studienrichtung wurde überprüft. Dabei zeigten sich auffällige C-Wert-Unterschiede zwischen Studierenden verschiedener Studiengänge (siehe Tabelle 2), aber sowohl für den Gesamteinfluss der Studienrichtung, als auch bei der Einzelprüfung der verschiedenen Studiengänge wurde kein statistisch signifikanter Regressionsparameter ermittelt ( $p>.05)$.

1 Die statistische Analyse der Daten zeigte, dass 53.5 Prozent der Varianz des C-Wertes durch das Ausmaß der dogmatisch religiösen Erziehung in der Kindheit erklärt werden $\left(R^{2}=.535\right)$. Durch den Regressionskoeffizienten in Höhe von 874 wurde deutlich, dass bei einem Anstieg der religiösen Erziehung um einen Punktwert der C-Wert um .874 Punktwerte steigt. Daraus folgt, dass hohe Werte in der Erziehungsskala, die mit einer nicht religiösen Erziehung einhergehen, die Moralkompetenz erhöhen, während niedrige Werte, die eine dogmatisch religiöse Erziehung implizieren, die C-Wert-Höhe vermindern. 


\begin{tabular}{|l|l|l|l|l|l|l|l|}
\hline \multicolumn{2}{|c|}{} & \multicolumn{6}{|l|}{ Moralische Kompetenz (C-Wert) } \\
\cline { 3 - 9 } \multicolumn{1}{|c|}{} & $n$ & $M$ & $S D$ & Mdn & Min & Max \\
\hline \multirow{4}{*}{$\begin{array}{l}\text { Studien- } \\
\text { richtung }\end{array}$} & Rechtswissenschaften & 5 & 13.67 & 15.94 & 10.05 & .91 & 41.33 \\
\cline { 2 - 9 } & Medizin und Pharmazie & 18 & 25.59 & 18.55 & 23.84 & 2.72 & 57.21 \\
\cline { 2 - 9 } & Psychologie & 36 & 25.31 & 18.16 & 24.87 & .33 & 68.37 \\
\cline { 2 - 9 } & Wirtschaftswissenschaften & 30 & 19.81 & 19.79 & 12.37 & .00 & 73.24 \\
\cline { 2 - 9 } & Naturwissenschaften & 6 & 15.28 & 19.91 & 8.52 & 1.92 & 55.47 \\
\cline { 2 - 9 } & Sozialwissenschaften & 13 & 15.33 & 12.37 & 14.83 & 3.62 & 47.71 \\
\cline { 2 - 9 } & Erziehungswissenschaften & 5 & 32.23 & 16.83 & 22.31 & 17.30 & 55.82 \\
\cline { 2 - 9 } & Technikwissenschaften & 5 & 15.36 & 14.03 & 13.05 & 2.16 & 39.01 \\
\cline { 2 - 8 } & Geisteswissenschaften & 3 & 10.25 & 11.94 & 3.41 & 3.30 & 24.04 \\
\hline
\end{tabular}

Tabelle 2.

Höhe der moralischen Kompetenz bei Studierenden diverser Studienrichtungen

Die im Rahmen dieser Studie gefundenen Unterschiede zwischen verschiedenen Studienrichtungen sind aufgrund der geringen und in den einzelnen Fachgruppen variierenden Anzahl der Befragten mit Vorsicht zu interpretieren. Dennoch sollte in künftigen Untersuchungen der Einfluss verschiedener Studienfächer auf die Moralkompetenz, insbesondere im Hinblick auf das Ausmaß der Gelegenheit zur Verantwortungsübernahme, untersucht werden.

Insgesamt konnte die erste Annahme, dass die dogmatisch religiöse Erziehung in der Kindheit die starken C-Wert-Differenzen der Studierenden erklärt, durch das Ausschließen anderer intervenierender Variablen bekräftigt werden.

Die Ergebnisse dieser Studie lassen einen ungewöhnlich starken Effekt von dogmatisch religiöser Erziehung auf die Höhe der Moralkompetenz festhalten: Studierende, die eine dogmatisch religiöse Erziehung in ihrer Kindheit berichten, weisen eine sehr viel geringere Moralkompetenz (8.59 C-Punkte) auf als Studierende, die eine nicht religiöse Erziehung berichten (40.41 C-Punkte). Dieser entwicklungshemmende Effekt von dogmatisch religiöser Erziehung wird durch eine Differenz von 31.82 C-Punkten zwischen beiden Gruppen unterstrichen (siehe Abbildung 1). 


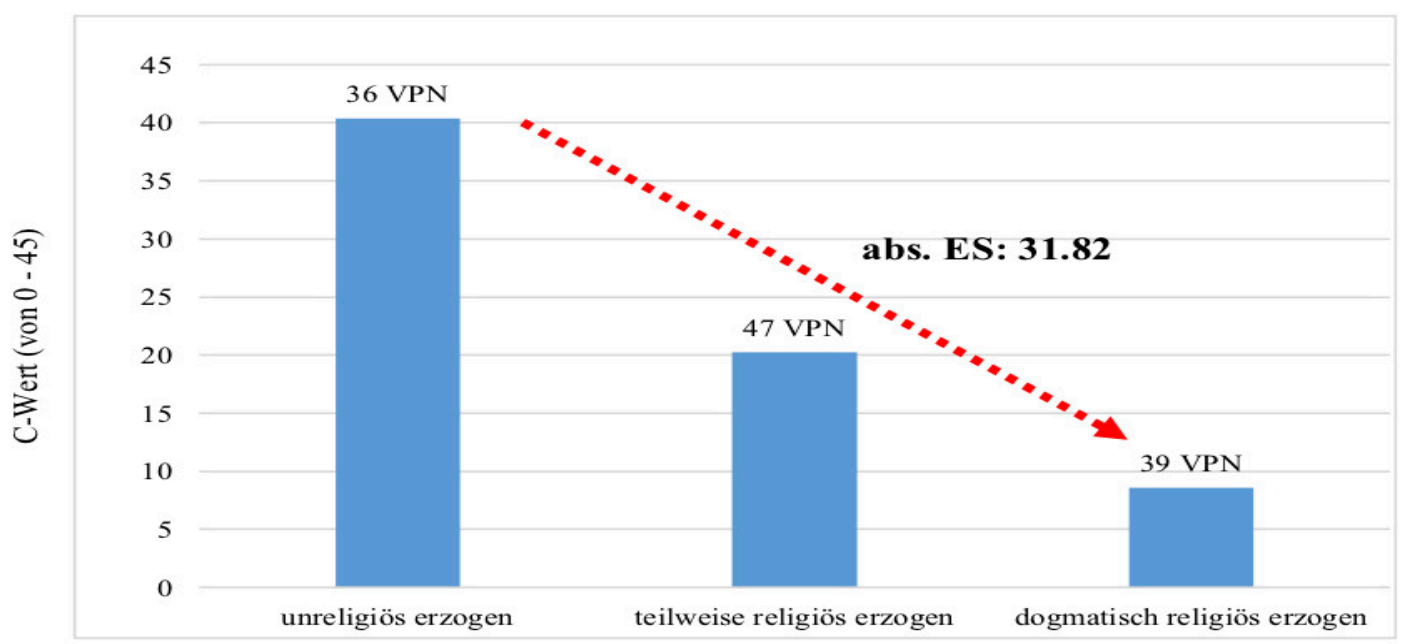

Ausmaß der religiösen Erziehung

Abbildung 1

Durchschnittliche C-Werte nicht religiös, teilweise religiös und dogmatisch religiös erzogener Probanden

Basierend auf dem gefundenen kompetenzhemmenden Einfluss einer dogmatisch religiösen Erziehung wurde zusätzlich geprüft, welche Einzelaspekte der religiösen Erziehung die verringerte Entwicklung der Moralkompetenz bedingen. Dazu wurde untersucht, wie die vier Unterskalen der religiösen Erziehung mit der C-Wert-Höhe zusammenhängen.

Die Allgemeine Relevanz der Religion, die religiöse Wertevermittlung, die religiösen Praktiken sowie die Reflexionsmöglichkeiten in der Erziehung hängen sehr hoch mit der Moralkompetenz zusammen $(r=.667-.710, p<.001)$. Auch hier konnte durch die Bestimmung der absoluten Effektstärken das praktische Ausmaß der Zusammenhänge verdeutlicht werden (siehe Abbildung 2-5).

Der größte Unterschied zeigte sich in Bezug auf die religiöse Wertevermittlung in der Kindheit: Studierende, die ein hohes Ausmaß an religiöser Wertevermittlung erfahren haben unterscheiden sich um 30.42 C-Punkte von Studierenden, denen kaum religiöse Werte vermittelt wurden. 




Ausmaß der religiösen Wertevermittlung

Abbildung 2. Durchschnittliche C-Werte von Studierenden mit kaum, teilweise und hoher religiöser Wertevermittlung in der Kindheit.

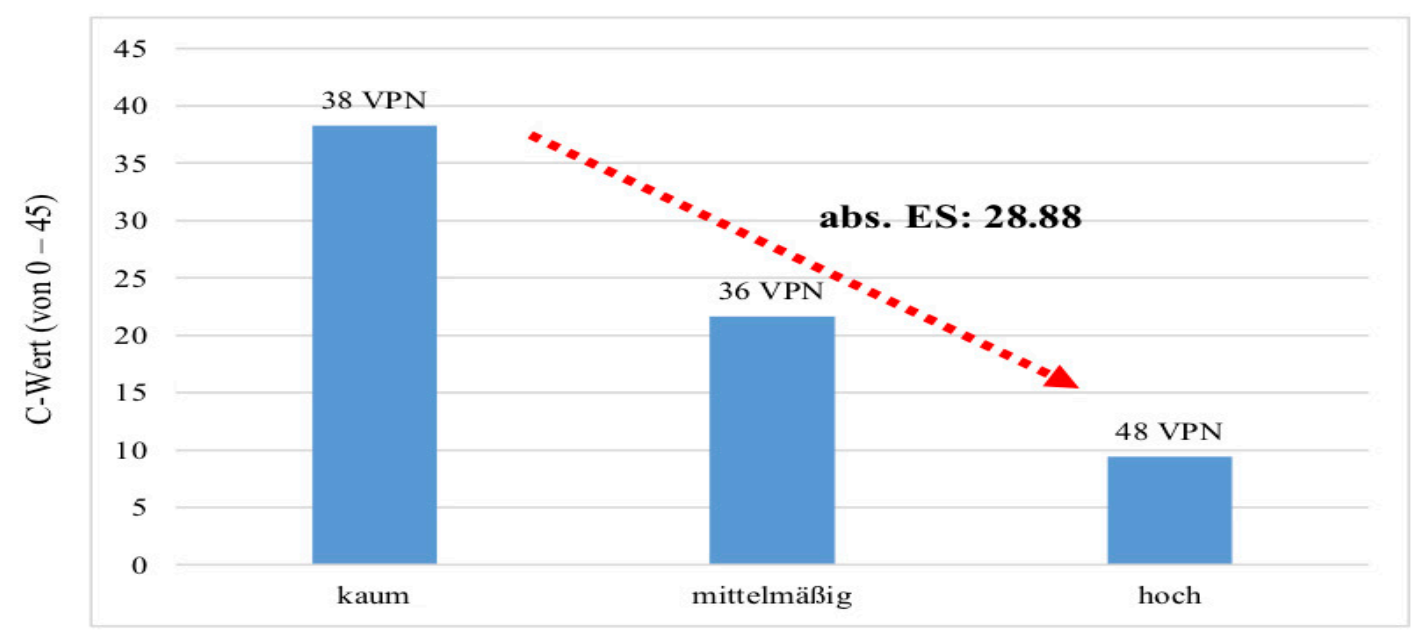

Ausmaß der Vermittlung religiöser Praktiken

Abbildung 3. Durchschnittliche C-Werte von Studierenden, die mit kaum, mittelmäßig angeleiteten und hochausgeprägten religiösen Praktiken in der Kindheit erzogen wurden. 


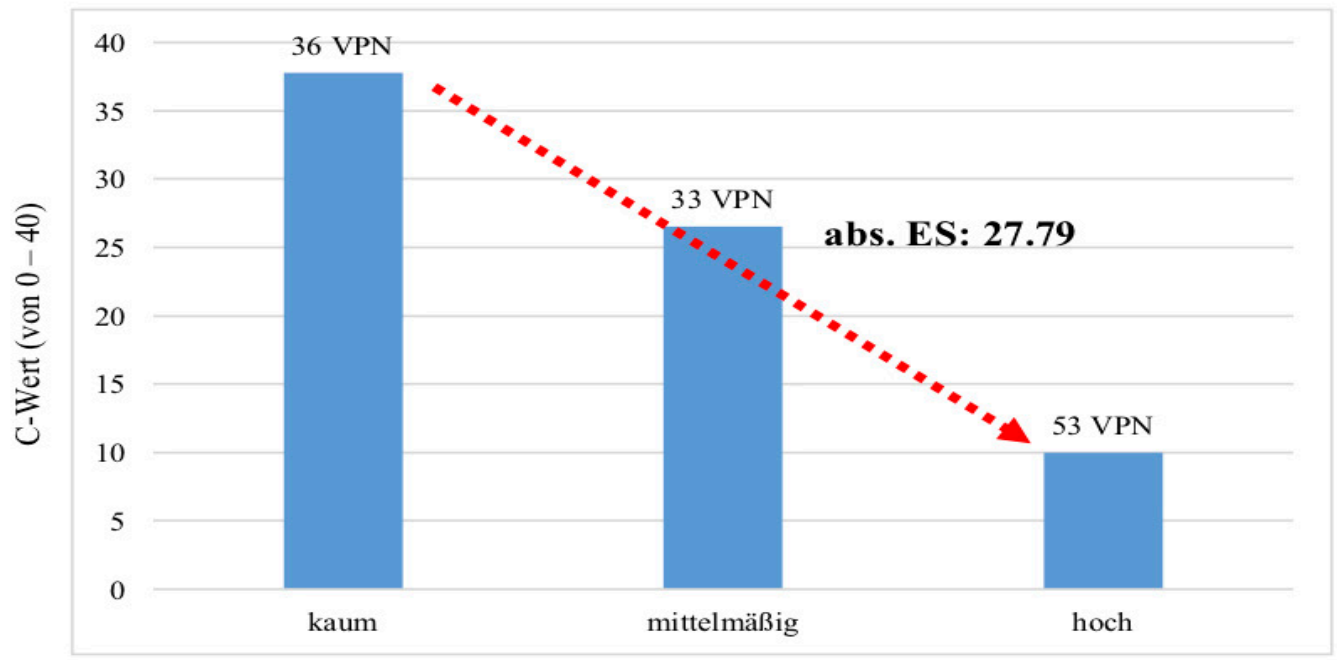

Relevanz der Religion in der Erziehung

Abbildung 4. Durchschnittliche C-Werte von Studierenden, die eine geringe, mittelmäßige und hohe Relevanz der Religion in der frühkindlichen Erziehung erfuhren.

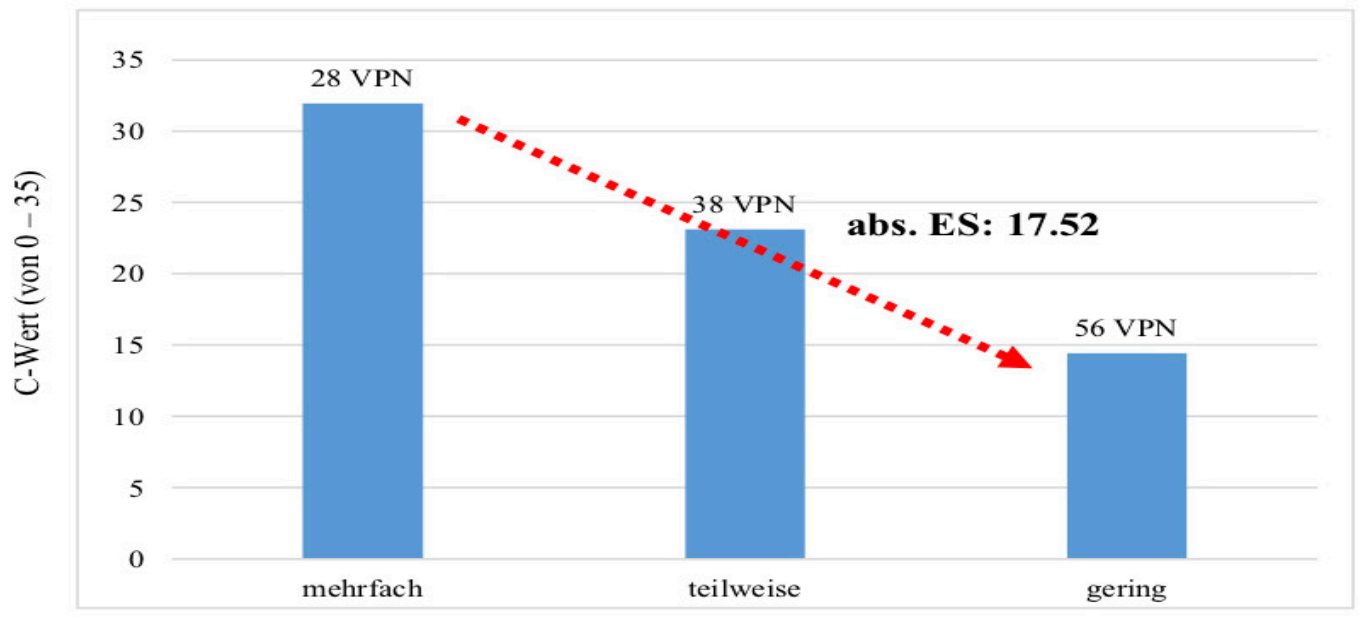

Ausmaß der Reflexionsmöglichkeiten in der Kindheit

Abbildung 5. Durchschnittliche C-Werte von Studierenden, die in der Kindheit mehrfache, teilweise und geringe Reflexionsmöglichkeiten in Bezug auf Religionen erfuhren.

Zur Prüfung der zweiten Hypothese, dass eine dogmatisch religiöse Erziehung in der frühen Kindheit zu einer erhöhten Präferenz niedriger (bzw. präkonventioneller) Stufen der Moralentwicklung führt, wurden für alle 122 Probanden sechs Mittelwerte für die jeweiligen sechs Orientierungstypen gebildet. Anschließend wurde untersucht, inwieweit sich dogmatisch religiös, teilweise religiös und nicht religiös erzogene Personen innerhalb der einzelnen Orientierungstypen signifikant voneinander unterscheiden.

Erste Erkenntnisse konnten durch die graphische Darstellung der arithmetischen Mittelwerte gewonnen werden: 


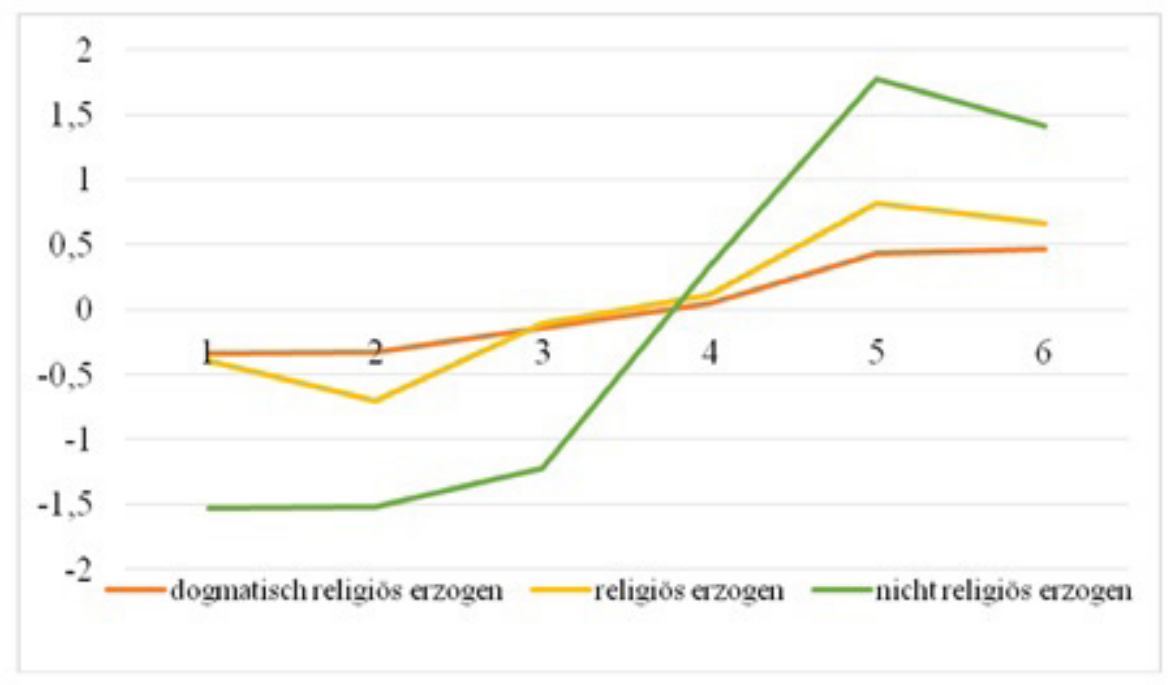

Abbildung 6. Präferenz der sechs moralischen Orientierungstypen bei dogmatisch religiös, teilweise religiös und nicht religiös erzogenen Probanden.

Die Abbildung bestätigt einerseits die von Lind (2015) postulierte universelle Konstanz im aufsteigenden Akzeptanzmuster und zeigt andererseits auffällige Abweichungen in der moralischen Orientierung der nicht religiös erzogenen Personen gegenüber den anderen Gruppen. Nicht religiös erzogene Studierende lehnen Typ eins bis drei (präkonventionelles Moralniveau) stärker ab und präferieren häufiger Typ fünf und sechs (postkonventionelles Moralniveau). Im Vergleich dazu akzeptieren dogmatisch religiös und teilweise religiös erzogene Probanden viel häufiger Typ eins, zwei und drei. Die Gruppenunterschiede bezüglich der moralischen Orientierungen erwiesen sich, außer in Typ vier, als statistisch signifikant $(\mathrm{p}<.001)$.

\section{Diskussion und Ausblick}

Religion gilt bis heute vorherrschend als Richtmaß für Moral (Ratzinger 1975). Religiöse Erziehung wird demnach als notwendige und förderliche Bedingung für die Moralentwicklung erachtet. Die hier vorgestellte Studie lässt diese Annahme anzweifeln: Die Daten zeigen, dass gerade Menschen, die eine dogmatisch religiöse Erziehung erfahren haben, eine sehr geringe Moralkompetenz aufweisen, und zwar eine sehr viel geringere als Menschen, die von einer nicht religiösen Erziehung in ihrer Kindheit berichten. Umso stärker die religiöse Verbindlichkeit in der frühkindlichen Erziehung war, desto geringer fiel die moralische Kompetenz der Studierenden im Erwachsenenalter aus. Dieser durchgreifende Einfluss der Erziehungserfahrung auf die Moralentwicklung zeigte sich zudem darin, dass selbst dogmatisch religiös erzogene Studierende, die sich von der 
Religion gelöst haben, ebenfalls eine geringe Moralkompetenz entwickelt hatten.

Die vorliegenden Daten bestätigen die Vermutung eines moralhinderlichen Einflusses der dogmatisch religiösen Erziehung. Die dogmatische Vermittlung einer Religion, die sich jeglicher Gegenauffassungen entzieht und eine nicht anzweifelbare Einstellung fordert, kann in der Erziehung von Kindern nachhaltige Folgen haben. Aus der dogmatischen Unterwerfung an eine Religion scheint eine Unfähigkeit zur selbstbestimmten und reflexiven Begründung moralischer Prinzipien zu resultieren. Wenn Eltern Denken und Diskussion über wichtige Fragen des Lebens unter Hinweis auf religiöse Dogmen („Das darf man nicht sagen! Das darf man nicht einmal denken! Gott sieht alles!" usw.) unterbinden, fehlt Kindern jegliche Stimulation ihres eigenen Denk- und Diskussionsvermögens. Die Entwicklung von Moralkompetenz braucht nach Lind (2015) Gelegenheiten zur Verantwortungsübernahme und angeleiteten Reflexion. Gerade diese Gelegenheiten scheinen den dogmatisch religiös erzogenen Studierenden im Elternhaus gefehlt zu haben: Während Studierende mit geringfügigen Reflexionsmöglichkeiten in der Kindheit einen durchschnittlichen C-Wert von 14.45 erreichten, zeigten Studierende mit mehrfachen Reflexionserfahrungen im Elternhaus eine hohe bis sehr hohe Moralkompetenz von durchschnittlich 31.97 C-Punkten.

Insbesondere der in der Studie hervortretende hohe negative Einfluss der religiösen Wertevermittlung lässt vermuten, dass die strikte und verbindliche Einhaltung religiös vorgeschriebener Werte in der Kindheit so stark internalisiert wird, dass eine Hinterfragung dieser im Erwachsenenalter erschwert ist. Dieser negative Zusammenhang lässt die grundsätzliche Bedeutung der sogenannten Werteerziehung in fragestellen. Der heutzutage immer häufiger diskutierte „Werteverfall“ und „Wunsch nach traditionellen handlungsleitenden Idealen“ (Bundesforum Familie 2008; Schubarth 2010; Standop 2005 ) ist aufgrund der aus der Studie hervorgehenden moralhemmenden Wirkung einer wertevermittelnden Erziehung anzuzweifeln. Wie bereits Lind (2015) betonte, scheint „die Vermittlung von Werten, worunter man oft ein Gemisch aus moralischen Prinzipien, religiös-kulturellen Überzeugungen und individuellen Präferenzen versteht, (...) meist wirkungslos, manchmal sogar schädlich“ (cf. 27) zu sein.

Der Vergleich der moralischen Orientierungen an den sechs Typen bestätigt die Annahme der modernen Moralpsychologie, dass moralische Orientierungen universell (und vermutlich angeboren) sind (Lind 2015). Obgleich die Teilnehmer - mit oder ohne dogmatisch religiöser Erziehung - sich sehr stark bezüglich ihrer Moralkompetenz unterschieden, zeigen sie dieselbe Präferenz für hohe Typen der Moralorientierung (Typ fünf und sechs) und dieselbe Ablehnung von niedrigen Typen (Typ eins und zwei).

Die Studie belegt zudem die moralpsychologische Grundannahme der kognitivaffektiven Parallelität: Je höher die Moralkompetenz einer Person ist, umso stärker orientiert sie sich an moralischen Prinzipien im engeren Sinne (Typ fünf und sechs), und umso mehr weist sie Argumentationen auf niedrigen Orientierungsebenen (Typ eins und zwei) zurück. Auch dieser in vielen Studien belegte Zusammenhang unterstreicht die 
Wichtigkeit der Förderung von Moralkompetenz. Die affektive Bindung an Moralprinzipien scheint den Menschen angeboren zu sein; sie muss nicht besonders gefördert werden. Die Fähigkeit, diese Prinzipien im Alltag anzuwenden und die Dilemmata zu lösen, in die man dabei unvermeidlich gerät, muss jedoch erlernt und gefördert werden.

Zusammengefasst deuten die vorgestellten Befunde an, dass moralische Fähigkeiten nur durch eine undogmatische und von jeglichen Religionen losgelöste Erziehung gefördert werden können. Eine explizite Untersuchung zu moralförderlichen Erziehungsmaßnahmen steht folglich noch an.

Über die künftige Umstrukturierung der elterlichen Moralerziehung hinaus stellt sich abschließend die Frage, wie und ob junge Erwachsene mit einem dogmatisch religiösen Hintergrund in ihren moralischen Fähigkeiten gefördert werden können. Dies stellt eine Herausforderung für die Hochschuldidaktik dar. Eine effektive und nachhaltige Möglichkeit zur Förderung der moralischen Kompetenz bietet die Konstanzer Methode der Dilemma-Diskussion (KMDD) nach Lind (2015). Die Prinzipien der Konstanzer Methode der Dilemma-Diskussion wurden in ihrer Wirksamkeit empirisch erwiesen und gelten als sehr effektiv und nachhaltig.

Die Stichprobe der vorgestellten Studie betrifft junge Erwachsene, die als zukünftige Eltern die Moralerziehung der nachfolgenden Generationen verantworten. Wenn es gelingt, ihre Moralkompetenz deutlich zu fördern, bestehen sie ihren späteren Kindern gegenüber, so kann man vermuten, auch weniger auf dogmatischer Wertevermittlung, sondern lassen ihnen mehr Raum zu eigenem Denken und Diskutieren. Wie die Längsschnittstudie von Speicher (1994) zeigte, ist die Fähigkeit der Eltern, mit ihren Kindern offen über Probleme zu diskutieren, ausschlaggebend für deren Moralentwicklung.

InsgesamtkonntendieErgebnissedervorgestelltenStudiedenbedeutendenEinfluss der frühkindlichen Erziehungserfahrungen für die Moralkompetenz im Erwachsenenalter belegen. Es lässt sich festhalten, dass dogmatisch religiöse Erziehungsmethoden als moralhemmend einzustufen sind, aber welche Erziehungsmethoden eine hohe Moralkompetenz fördern bleibt folglich unbeantwortet und bedarf einer ausführlichen Untersuchung.

\section{Literatur}

Adorno T. W. 1950. The Authoritarian Personality. New York: Harper.

Albani C., Bailer H., Blaser G., Geyer M., Brähler E., \& Grulke N. 2002. “Erfassung religiöser und spiritueller Einstellungen". PPmP: Psychotherapie Psychosomatik Medizinische Psychologie 52(07):306-13. 
Bundesforum Familie (Hrsg.) 2008. Elternstimmen. Vorbilder und Werte in der Erziehung. Zwei Studien des Bundesforums Familie und des Kindersenders NICK. Berlin: Bundesforum Familie.

Figl J. 2017. Handbuch Religionswissenschaft: Religionen und ihre zentralen Themen. Innsbruck: Tyrolia.

Fromm E. 2014. Die Furcht vor der Freiheit. Escape from Freedom: Open Publishing Rights GmbH.

Gruen A. 2000. Der Fremde in uns. Klett-Cotta DTV.

Glover R. J. 1997. "Relationships in Moral Reasoning and Religion Among Members of Conservative, Moderate, and Liberal Religious Groups". The Journal of Social Psychology 137(2):247-54.

Haumann W. 2006. Generationen-Barometer 2006: eine Studie des Instituts für Demoskopie Allensbach. Freiburg: Karl Alber.

Holley R. T. 1991. „Assessing Potential Bias: The Effects of Adding Religious Content to the Defining Issues Test". Journal of Psychology and Christianity 10:323-36.

Huber S. 2009. "Der Religionsmonitor 2008: Strukturierende Prinzipien, operationale Konstrukte, Auswertungsstrategien". Woran glaubt die Welt. Gütersloh: Bertelsmann Stiftung (17-52).

Jakobs M. 2006. "Religiosität als biografische Verarbeitung von Religion”. Religiosität: Anthropologische, Theologische und Sozialwissenschaftliche Klärungen 116.

Kohlberg L. \& Althof W. 1996. Die Psychologie der Moralentwicklung. Frankfurt am Main: Suhrkamp.

Lind G. 2000. "The Importance of Responsibility-Taking Opportunities for Self-sustaining Moral Development." Journal of Research in Education 10(1):9-15.

Lind G. 2003. Moral ist lehrbar: Handbuch zur Theorie und Praxis moralischer und demokratischer Bildung. München: Oldenbourg.

Lind G. 2015. Moral ist lehrbar. Wie man moralisch-demokratische Kompetenz fördern und damit Gewalt, Betrug und Macht mindern kann. Berlin: Logos.

Lind G. 2016. "Die Bedeutsamkeit empirischer Befunde: Statistische Signifikanz vs. relative vs. absolute Effektstärken". Abgerufen von https://www.uni-konstanz.de/ ag-moral/pdf/Lind-2016_Effektstaerke-Vortrag.pdf

Lind G. 2018. "Reporting the C-score”. Abgerufen von https://www.uni-konstanz.de/agmoral/mut/_Reporting_the_C_score.pdf

Lupu I. 2009. Moral, Lernumwelt und Religiosität: Die Entwicklung moralischer Urteilsfähigkeit bei Studierenden in Rumänien in Abhängigkeit von Verantwortungsübernahme und Religiosität. (Dissertation), Universität Konstanz.

Maiello C. 2007. Messung und Korrelate von Religiosität. Münster: Waxmann.

Meulemann H. 2015. Nach der Säkularisierung. Religiosität in Deutschland 1980-2012.

Wiesbaden: Springer Fachmedien.

Ratzinger J. 1975. Prinzipien christlicher Moral. Einsiedeln: Johannes Verlag. 
Richards P. S. 1991. "The Relation between Conservative Religious Ideology and Principled Moral Reasoning: A Review". Review of Religious Research 359-68.

Saeidi-Parvaneh S. 2011. Moral, Bildung und Religion im Iran: Zur Bedeutung universitärer Bildung für die Entwicklung moralischer Urteils- und Diskursfähigkeit in einem religiös geprägten Land. (Dissertation), Universität Konstanz.

Schillinger M. 2006. Learning Environment and Moral Development: How University Education Fosters Moral Judgment Competence in Brazil and two German-speaking Countries. Aachen: Shaker.

Schubarth W. 2010. „Die »Rückkehr der Werte«. Die neue Wertedebatte und die Chancen der Wertebildung". In W. Schubarth, K. Speck, \& H. L. von Berg (Hrsg.), Wertebildung in Jugendarbeit, Schule und Kommune. Wiesbaden: VS Verlag für Sozialwissenschaften (21-41).

Speicher B. 1994. "Family Patterns of Moral Judgment During Adolescence and Early Adulthood." Developmental Psychology 30:624-32.

Standop J. 2005. Werte-Erziehung. Einführung in die wichtigsten Konzepte der Werteerziehung (Studientexte für das Lehramt, 18). Weinheim u.a.: Beltz. 
Asli Akin (Berlin)

Religiöse Erziehung und Moralentwicklung:

Der Einfluss einer religiös geprägten Kindheit auf die moralische

Orientierung und Kompetenz

Zusammenfassung: Die bisherige moralpsychologische Forschung zeigt, dass Bildungseinrichtungen einen entscheidenden Einfluss auf die Entwicklung der moralischen Orientierung und Kompetenz haben. In der vorgestellten quantitativen Untersuchung wurde der Einfluss der frühkindlichen Erziehungserfahrungen auf die moralischen Fähigkeiten erforscht. Da die Moralerziehung von Kindern häufig mit religiösen Normen und Werten verbunden wird, und Religionen aktuell in ihrer Bedeutung und Funktionalität im öffentlichen Diskurs stehen, wurde speziell die religiöse Moralerziehung in ihrer Wirkung auf die moralische Entwicklung empirisch ergründet. Dazu wurden Studierende verschiedener Fachrichtungen aus Berlin zur religiösen Prägung in ihrer frühkindlichen Erziehung befragt und in Bezug auf ihre moralische Orientierung und Kompetenz untersucht. Den messmethodischen Rahmen bilden der Moralische-Kompetenz-Test nach Lind und der selbstkonstruierte sowie im Voraus pilotierte Fragebogen zur Erfassung der religiösen Erziehung und Religiosität. Die Auswertung der Online-Studie konnte zeigen, dass die moralischen Fähigkeiten bei dogmatisch religiös erzogenen Studierenden signifikant vermindert ausfallen und dabei nicht auf unterschiedliche Bildungserfahrungen oder das gegenwärtige Beibehalten der Religiosität zurückzuführen sind.

Schlüsselbegriffe: religiöse Erziehung; Moralentwicklung; moralische Kompetenz; moralische Orientierung; Studierende 


$$
\text { Asli Akin (Berlin) }
$$

\title{
Religious Education and Moral Development:
}

\section{The influence of a religious childhood on moral orientation and competence}

\begin{abstract}
The previous moral psychological research on educational institutions highlighted the influence on the development of moral orientation and competence. With the help of the present quantitative study, the influence of early childhood education on moral abilities has been explored. Since the moral education of children is often associated with religious norms and values, and religions are debated in relation to their general meaning and functionality, the religious moral education has been investigated. Therefore, students of various disciplines from Berlin were asked about their religious education in their early childhood and were examined on their current moral orientation and competence. The Moral-Competence-Test by Lind and a self-constructed and piloted questionnaire for the examination of religious education and religiosity have been used for the measurement. The results of the online study have shown that the moral skills of dogmatically educated students are significantly reduced, but not related to the differences in educational experience or the current preservation of religiousness.
\end{abstract}

Keywords: religious education; moral development; moral competence; moral orientation; university students

Ethics in Progress (ISSN 2084-9257). Vol. 9 (2018). No. 2, Art. \#3, pp. 27-43.

Creative Commons BY-SA 3.0

Doi:10.14746/eip.2018.2.3 